\title{
Umbilical Cord Perivascular Cells: A Mesenchymal Cell Source for Treatment of Tendon Injuries
}

\author{
Hamideh Emrani and John E. Davies*
}

Faculty of Dentistry and Institute of Biomaterials and Biomedical Engineering, University of Toronto, 164 College Street, Toronto, Ontario, M5S 3G9, Canada

\begin{abstract}
Objective: To model the healing that umbilical cord perivascular cells effect in equines, we have used human umbilical cord perivascular cells (HUCPVCs) to repair tendon damage in a collagenase tendon injury model in immunecompromised rats. Animals: 48 Nude rats (Crl:NIH-Foxn $\left.1^{\mathrm{rnu}}\right)$ of 200-250 g weight were used. Procedure: The Achilles tendon was exposed by blunt dissection and, using a $30 \mathrm{G}$ needle, $30 \mu \mathrm{l}$ of either a mixture of cells $\left(2 \times 10^{5}\right)$ and collagenase, or collagenase alone, was injected close to the musculotendinous junction in the direction of the osteotendinous junction. Results: Harvested tendons showed the presence of HUCPVCs at the site of injury, whose morphology changed from ovoid to elongated over the 30 post-injury experimental time period. Human genes for collagen type 1 and $\beta$-actin were expressed at all time points and there was also a significant increase in tendon tensile strength and stiffness by 30 days post-injury in the experimental group. Conclusion: HUCPVCs facilitated regeneration in our model through changes in collagen organization; cell shape and orientation; and increase in mechanical properties over those of the untreated controls. Clinical Relevance: Cell therapy has been shown to be effective in treating tendon injuries, especially in equines. We have recently isolated a mesenchymal cell population from equine umbilical cords that is analogous to a well-characterized HUCPVC population. Our results indicate that HUCPVCs are a putative cell source for treating tendon injuries; and this model could explain the benefits of using analogous cells in equines.
\end{abstract}

Keywords: Umbilical cord, HUCPVC, Tendon repair, Collagenase injury model, Rat, Achilles tendon, Regeneration, Tensile strength.

\section{INTRODUCTION}

Injuries to tendon and ligaments are the cause of substantial morbidity for humans and other species especially horses. When a tendon is damaged the repair mechanism results in a "haphazard" healing [1] that forms a scar tissue within the tendon, which in turn decreases the overall elasticity in the damaged section of the tendon and increases strain on adjacent uninjured tissue. Therefore, there is a need for treatment methods that regenerate tissue in injuries for which natural repair mechanisms do not deliver functional recovery.

Cellular therapy has been the focus of many studies as a putative method for effectively treating a range of mammalian connective tissue disorders. Indeed, bone marrow derived cells [2], heterogeneous populations of cells derived from enzymatically digested fat tissue [3], and umbilical cord blood [4] have all been used in treating tendon injuries in horses; as recently reviewed by Frisbie and Smith [5]. Plastic adherent bone marrow-derived cells, commonly referred to as bone marrow stem, or stromal, cells (BMSC) have been reported to successfully heal tendons, particularly superficial digital flexor tendon injuries in racehorses [3-6]. However, to facilitate the study of the fate and efficacy of such administered cells several small animal models have been employed. Thus, both autologous [7] and allogeneic [8]

*Address correspondence to this author at the Faculty of Dentistry and Institute of Biomaterials and Biomedical Engineering, University of Toronto, 164 College Street, Toronto, Ontario, M5S 3G9, Canada; Tel: 4169781471; Fax: 4169465639; E-mail: jed.davies@utoronto.ca
BMSCs have been shown to regenerate tissue in injured rabbit tendons, producing organized collagen fibers and resulting in higher tendon stiffness and modulus in the treatment groups compared to controls; although this cell source can also result in bone spicules at the injury site [8]. A rat patellar tendon injury model has also demonstrated improved strength and quality of repaired tissue with human BMSCs compared to human fibroblasts derived from tendon tissue [10].

We, and others, have described a rich source of human mesenchymal cells found in the perivascular region of the human umbilical cord [11-13] which, due to its very high CFU-F frequency, has enabled the first robust single cell clonal confirmation of a hierarchy of MSC differentiation [14], and which we have called human umbilical cord perivascular cells (HUCPVCs). While these cells exceed the minimum criteria established by the International Society for Cellular Therapy to define MSC [15], their default lineage is fibroblastic, making them ideal candidates for tendon tissue engineering. Like bone marrow derived stromal cells, these cells are both non-alloreactive and immunosuppressive [16] and thus suitable for allogeneic application. Indeed, allogeneic animal studies, as well as allogeneic human clinical trials have demonstrated the utility of MSCs without the need for tissue matching between donor and recipient. However, xenogeneic grafting of MSC would appear to be species dependent, and it has been reported that MSC cannot be employed to cross xenogeneic boundaries [17], which is why it is necessary to conduct human cell studies in immuno-compromised hosts. 
In the present work we wished to study the capacity of HUCPVCs to contribute to the healing of tendon injuries. To this end, we delivered HUCPVCs into tendon injuries in immuno-compromised rats within the context of modeling the healing that we have seen clinically when employing similar cells from equine umbilical cords to treat superficial digital flexor tendon injuries.

\section{MATERIALS AND METHODS}

\subsection{Cells}

Human Umbilical Cord Perivascular cells (HUCPVCs) frozen and stored in $1.8 \mathrm{ml}$ CryoTubes $^{\mathrm{a}}$ in liquid nitrogen at passage 2 , were provided by Tissue Regeneration Therapeutics Inc. (Toronto, ON). The cell vials were quickly thawed in a $43^{\circ} \mathrm{C}$ bath and $1 \mathrm{ml}$ of $\alpha$-MEM ${ }^{\mathrm{b}}$ was added. After leaving at room temperature for 2 minutes, the contents of the cell vials were transferred to a $15 \mathrm{ml}$ conical tube ${ }^{\mathrm{c}}$ the volume (2ml) was doubled with addition of $\alpha$-MEM and after 2 minutes incubation; additional $\alpha$-MEM was added to achieve a total volume of $10 \mathrm{ml}$, and the tubes were centrifuged at $285 \times \mathrm{xg}(1150 \mathrm{rpm})$ at $4^{\circ} \mathrm{C}$ for 5 minutes. The supernatant was removed and $1 \mathrm{ml}$ of $\alpha$-MEM was added to the cells. Cells were then counted using a Vicell automated cell counter ${ }^{c}$. The required volume of cells (see below) was centrifuged at $285 \mathrm{xg}(1150 \mathrm{rpm})$ at $4^{\circ} \mathrm{C}$ for 5 minutes and supernatant was removed, and bacterial collagenase type $\mathrm{I},{ }^{\mathrm{d}}$ dissolved in PBS and sterile filtered through a $0.22 \mu \mathrm{m}$ filter, was added to the cells. Then, the mixture was delivered on ice to the site of surgery.

\subsection{Surgical Protocol}

Nude rats (Crl:NIH-Foxn $1^{\text {rnu }}$ ) of 200-250 g were used. The surgery was a single injection of collagenase $(30 \mu \mathrm{l}$, $10 \mathrm{mg} / \mathrm{ml}$ ), or cells and collagenase, into the Achilles tendon. The collagenase injection method has been widely used to simulate a chronic tendon inflammation in rats $[18,19]$ and is considered an acceptable model in equines [20]. Under inhalation anesthesia (Isofluorane in nitrous oxide and oxygen, $900 \mathrm{ml}$ total flow rate; $5 \%$ induction and 2.0 to $2.5 \%$ maintenance), the skin overlying the right Achilles tendon was shaved and a longitudinal incision was made slightly medial to the visible outline of the tendon. The tendon was exposed by blunt dissection and, using a $30 \mathrm{G}$ needle, $2 \times 10^{5}$ cells in $30 \mu 1$ of collagenase ${ }^{\mathrm{d}}(10 \mathrm{mg} / \mathrm{ml})$ was injected close to the musculotendinous junction in the direction of the osteotendinous junction. The left Achilles tendon served as a sham control by injection of an equal volume of collagenase solution $(30 \mu 1,10 \mathrm{mg} / \mathrm{ml})$ without cells. For all groups, biodegradable sutures were used to close the incision.

Surgeries were performed on two groups of animals. One group was used for histological and molecular analysis of the tendon samples and the other group was used for mechanical testing studies. For the first 30 rats, time points were one day, 3 days, 7 days, 15 days, and 1 month post injection $(n=6$ for each time point). The second group of 18 rats, used for mechanical testing studies, had time points of 7 days, 15 days, and 1 month post injection ( $\mathrm{n}=6$ for each time point). Therefore, a total number of 48 animals were used for this experiment. Animals were euthanized by $\mathrm{CO}_{2}$ inhalation, and the Achilles tendons were excised and prepared according to the methods described below.

\subsection{Sample Preparation}

Samples for paraffin sectioning were fixed in Zinc-formalin [21] for 48 hours to one week, then transferred to $10 \%$ neutral buffer formalin for longer storage if necessary. The samples were rinsed in distilled water and dehydrated in an ascending series of ethanols for 30 minutes at each step. Then, the samples were immersed in methyl benzoate overnight and placed in toluene for 1 hour on a shaker. After this, samples were immersed in 3 changes of paraffin wax at $60^{\circ} \mathrm{C}$ in an oven under a $20 \mathrm{psi}$ vacuum for $1 \mathrm{hr}$ at each exchange. Samples were embedded using a Leica EG $1160^{\mathrm{e}}$ embedding station.

Since tendon is a highly fibrous tissue additional preparation was necessary for gaining optimal sections. Paraffin blocks were sectioned using a rotary microtome ${ }^{\mathrm{f}}$ to expose the tendon surface, then the block was immersed in ammonia for 10 minutes, wiped and immersed in Molliflex tissue softener for an additional 10 minutes and put in an ice water bath. This step facilitated the cutting of $6 \mu \mathrm{m}$ thick sections, which were then placed on APES coated slides and dried at $50^{\circ} \mathrm{C}$ overnight. Slides were dewaxed and dehydrated in xylene, and a descending series of ethanols, before staining.

\subsection{Immunohistochemistry}

Immunohistochemical detection of human cells was performed on paraffin sections. The primary antibody used was monoclonal mouse anti human nuclei ${ }^{\mathrm{g}}(1: 50, \mathrm{HuNu})$. Two indirect detection methods of the labeling were used. For some samples an avidin-biotin assay, using a biotinylated horse anti mouse IgG secondary antibody ${ }^{\mathrm{h}}$ and an avidin-linked peroxidase was employed with detection achieved through Nova $\mathrm{Red}^{\mathrm{h}}$. For some of these sections counterstaining was achieved with Mayer's hematoxylin for 10 minutes. For other sections, an Alexa Fluor assay, using a goat antimouse $\operatorname{IgG}$ secondary antibody conjugated with Alexa Fluor 700 was employed. These sections were counterstained with Hoechst or DAPI (1:1000 dilution) for 20 minutes and mounted with Fluoromount ${ }^{\mathrm{d}}$. In addition, activated macrophages in the defect site were identified using a monoclonal mouse anti-rat CD68 antibody ${ }^{i}$ (1:50). These sections also served as isotype controls. For each method some sections were prepared without addition of the primary antibody to serve as secondary controls. Collagen immunostaining was performed using a rabbit anti-human collagen type I polyclonal antibody ${ }^{j}$ and horseradish peroxidase; this antibody cross-reacts with human and rat collagen type I.

\subsection{Reverse Transcription (RT)-PCR}

\subsubsection{RNA Isolation}

In order to check for gene expression, tendon samples were immediately frozen in liquid nitrogen, ground to a powder, and stored at $80^{\circ} \mathrm{C}$ in $1.5 \mathrm{ml}$ eppendorf tubes. In order to extract total RNA, an electronic homogenizer was used on the frozen samples, and $1 \mathrm{ml}$ Trizol (TRI) Reagent ${ }^{\mathrm{k}}$ was added. Samples were treated based on previously described protocol [1]. 


\subsubsection{Primer Design}

The Ensembl ${ }^{\mathrm{m}}$ and Primer3 websites and AmplifX (version 1.5 .4 by Nicolas Jullien) software were used to design the primers. The accuracy of a sequence was checked in PUBMED/Nucleotide, and in order to check for PCR products of the primers against the entire species, the BLAST program on PUBMED was referenced. A total of 20 primers were designed for collagen and the tenocyte genes tenomodulin, tenascin and decorin (the design of the primers is reported in full in Emrani [22]).The amplified PCR product was fractionated on a $1.2 \%$ agarose gel and visualized by ethidium bromide staining.

\subsection{Tendon Mechanical Testing}

After sacrifice, the rat Achilles tendons were dissected with the calcaneous attached. All muscle tissue was removed together with any residual soft tissue. The samples were then immersed in PBS and transferred for immediate mechanical testing. Before starting the test, each tendon was measured using a toolmaker's microscope. Specifically, the length and thickness of the tendon at the two ends, and the middle section of the tendon, were recorded and cross-sectional area of the tendon was calculated. An Instron $\mathrm{MTS}^{\mathrm{p}}$ was used to load the tendon samples in tension using a $1 \mathrm{kN}$ load cell $(10 \% \mathrm{~F}, 100 \mathrm{~N})$. The ends of the samples were secured into the grips of the MTS brass clamp and Hematoxylin stain lines were carefully placed perpendicular to the long axis of the tendon to mark the gauge length. The tendon was preconditioned through 3 cycles of $0.1-0.5 \mathrm{~N}$ at $10 \mathrm{~mm} / \mathrm{min}$. After preconditioning samples were tested to failure at $10 \mathrm{~mm} / \mathrm{min}$. Displacement and failure forces were recorded and mean ultimate strain was calculated as displacement at failure over initial tendon length (Mean Ultimate Strain (\%) $=($ Displacement $) /($ Initial Length $) * 100)$. Tensile strength was calculated as ultimate force at failure point over average cross sectional area of the tendon (Tensile strength = (Ultimate force)/(cross sectional area)). Additionally, at 30 days, the Young's Modulus was calculated.

\subsection{Statistical Analysis}

The non-parametric Wilcoxon Matched-Pairs SignedRank Test was used to assess the statistical significance of the difference in tendon tensile strength between samples that were injected with HUCPVCs in collagenase and the ones that received collagenase only. In all cases, $\mathrm{P}<0.025$ was taken to be significant.

\section{RESULTS}

\subsection{Immunohistochemistry}

Fig. (1) shows serial sections after being labeled using the avidin-biotin peroxidase enzyme system for $\mathrm{HuNu}$, without and with hematoxylin nuclear counterstaining, and CD68 respectively. When the Alexa 700 goat antimouse IgG was used as the secondary antibody the nuclei were counterstained with DAPI as shown in Fig. (2). HuNu labeled cells were visible in the sections in all time points. However, contrary to the product information, the label was seen in both nuclei and cytoplasm.
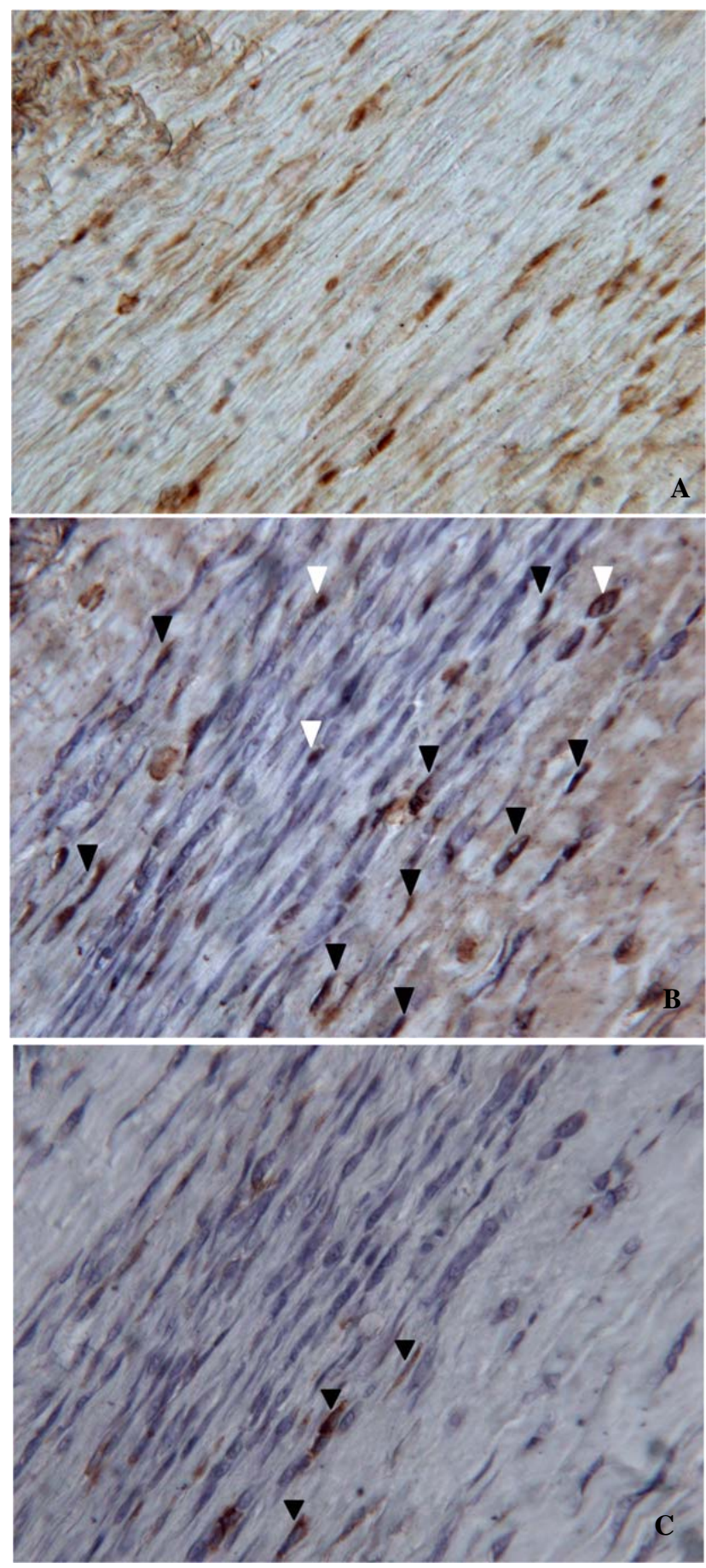

Fig. (1). Images of tendons 15 days post transplantation. The sections were labeled with anti human nuclei $(\mathrm{HuNu})$ primary antibody. (A) shows positively stained nuclei as brown spots inside rat tendon. (B) shows a serial section of (A) with hematoxylin counterstain. White triangles point to nuclei of cells that still have an ovoid morphology; black triangles represent more elongated cells. Activated macrophages are seen in $(\mathbf{C})$ the next serial section. Positive cells have dark brown nuclei $(\mathrm{FW}=343 \mu \mathrm{m})$. There were no areas that could be positively identified for both $\mathrm{HuNu}$ and antirat CD68 staining, indicating that the cells were not engulfed by macrophages. 

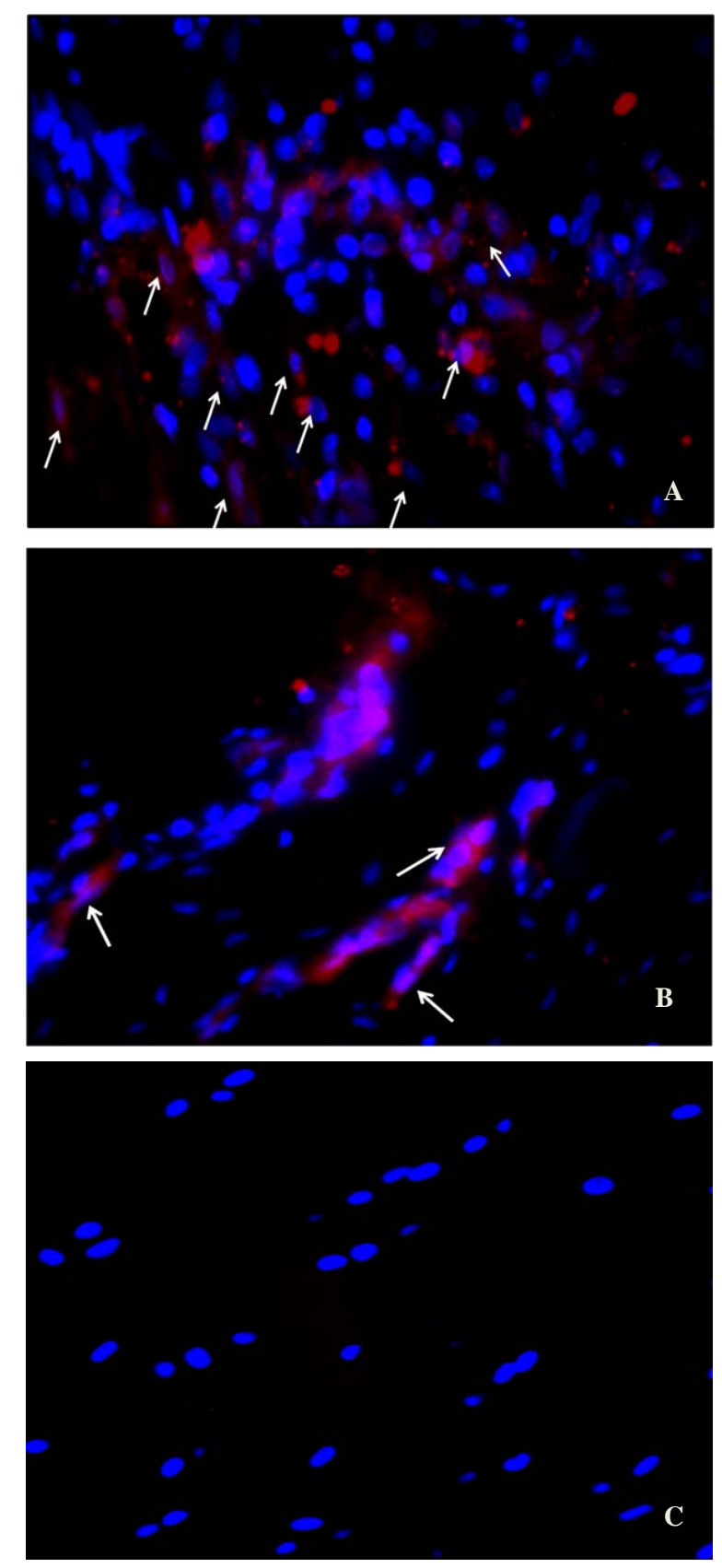

Fig. (2). (A) and (B) show positively stained cells within the tendon tissue 1 and 30 days post transplantation (white arrows). (C) Shows a section from the control group labeled with $\mathrm{HuNu}$, but lacking crossreactivity $(\mathrm{FW}=217.6 \mu \mathrm{m})$. The HUCPVC morphology of the cells has changed from ovoid at earlier time points into elongated at day 30 post surgery.

Interestingly, cells appeared more elongated and less ovoid with time, as can be seen in Fig. (2). Isotype controls were negative. Collagen type-1 immunolabeling showed the difference in density and alignment of the collagen fibers in the experimental and control groups. At earlier time points the collagen was randomly oriented in both groups (data not shown). However, by 30 days post treatment while the control samples showed no change in this random distribution (Fig. 3A) the experimental groups containing HUCPVCs clearly demonstrated a more linear fiber arrangement (Fig. 3B).
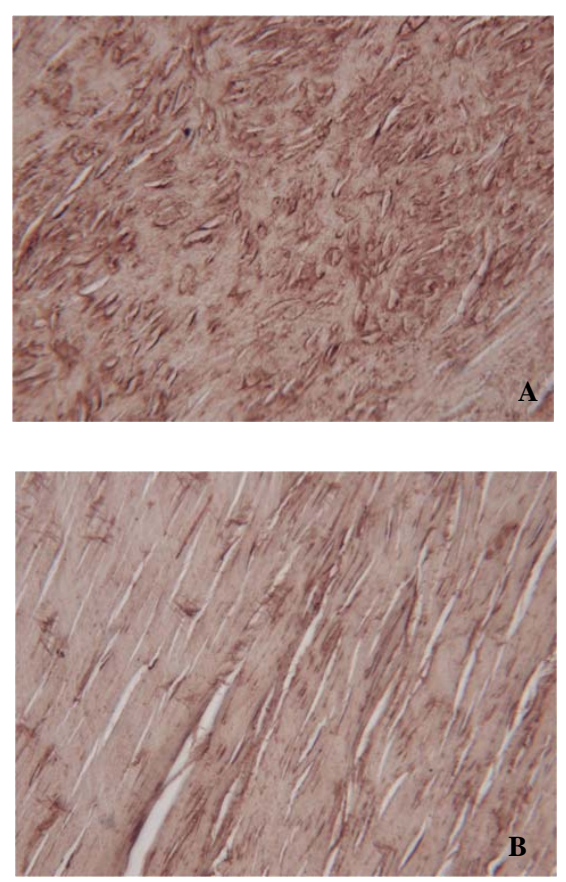

Fig. (3). Images of tendons 30 days post transplantation. The sections were labeled with anti collagen type-1 primary antibody. (A) Shows a tendon from the control group: this group did not receive HUCPVCs. (B) Shows a tendon from the experimental group. $(\mathrm{FW}=343 \mu \mathrm{m})$. The experimental group exhibits a more linear collagen fiber arrangement compared to the control group.

\subsection{PCR}

At all times points, differences in gene expression were only seen with the collagen type 1A1 primers employed. Additionally, expression of $\beta$-actin-5 - which is only expressed in human cells — was seen at all the time points; this indicated that the HUCPVCs survived in vivo until the 30 day experimental end point.

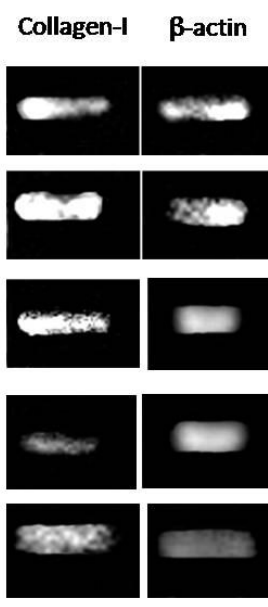

Rat Tendon +HUCPVC
Collagen-I $\beta$-actin

Day 1

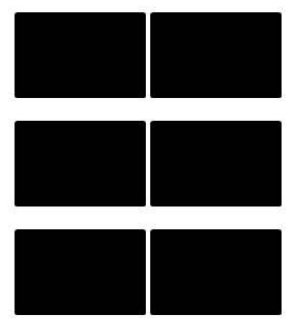

Day 15

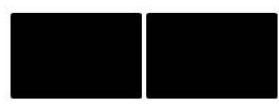

Day 30

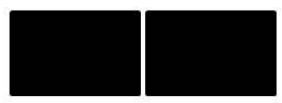

Rat Tendon
Fig. (4). RT-PCR of samples from 1, 3, 7, 15 and 30 days post surgery. $\beta$-actin and collagen-I showed clearly the difference between samples that contained HUCPVCs and the control samples. The presence of human collagen-I and the housekeeping gene, $\beta$-actin, show that the human cells were still alive at all time points. 


\subsection{Tendon Tensile Strength Test}

The HUCPVC-injected samples showed an increase in tensile strength when compared to the control group at the 30-day time point (Figs. 5 and $\mathbf{6}$ ). The overall mean tensile strength of the treatment group was $5.39 \pm 1.36 \mathrm{MPa}$, compared to $3.30 \pm 1.27 \mathrm{MPa}$ for the control group, which represented a $63.23 \%$ increase in strength. Non-parametric Wilcoxon Matched-Pairs Signed Rank statistical analyses revealed significant increases in both the tendon tensile strength and Young's modulus (stiffness) in the HUCPVC group $(\mathrm{P}<0.025)$.

\section{DISCUSSION}

Our data demonstrate that HUCPVCs facilitate healing of collagenase induced tendon injury in rats by expressing

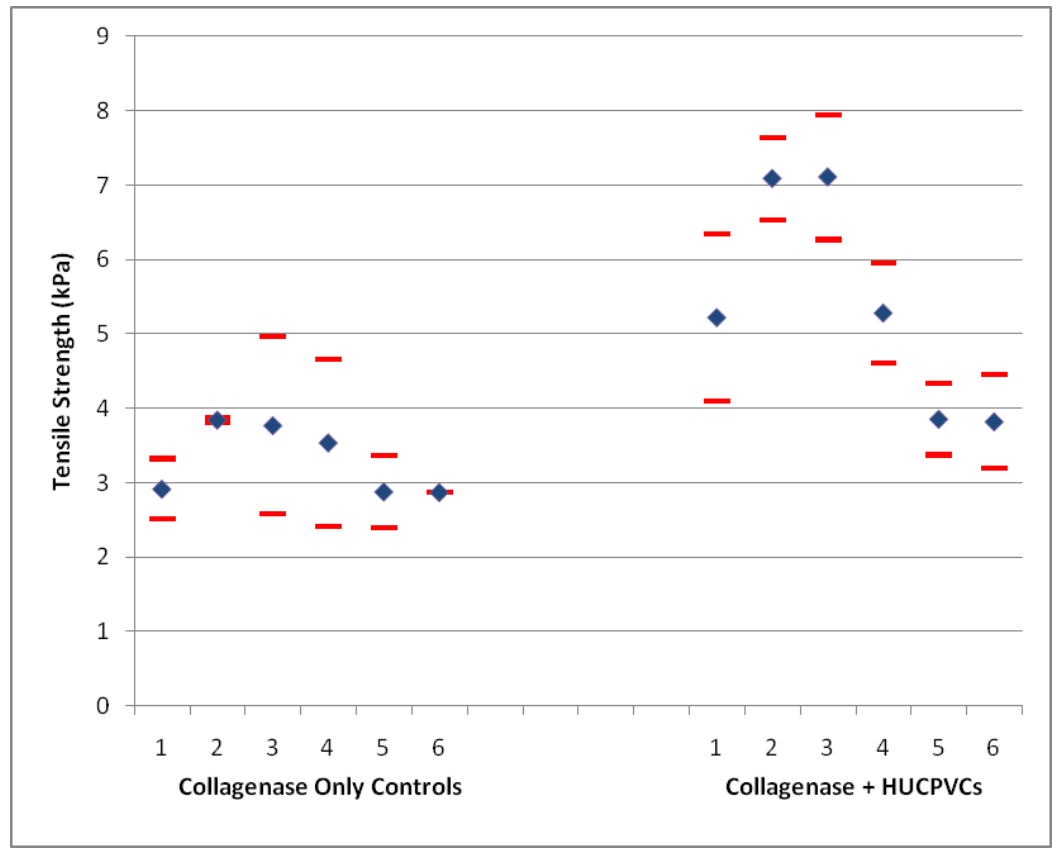

Fig. (5). Tendon tensile strength for 30 day post surgery samples of experimental and control groups $(\mathrm{n}=6)$. For each animal, blue diamonds represent mean tensile strength and pink bars represent single tensile measurements. As seen in the image, and shown by the statistical analysis, the group that received HUCPVCs had significantly higher tensile strength values compared to the control samples.

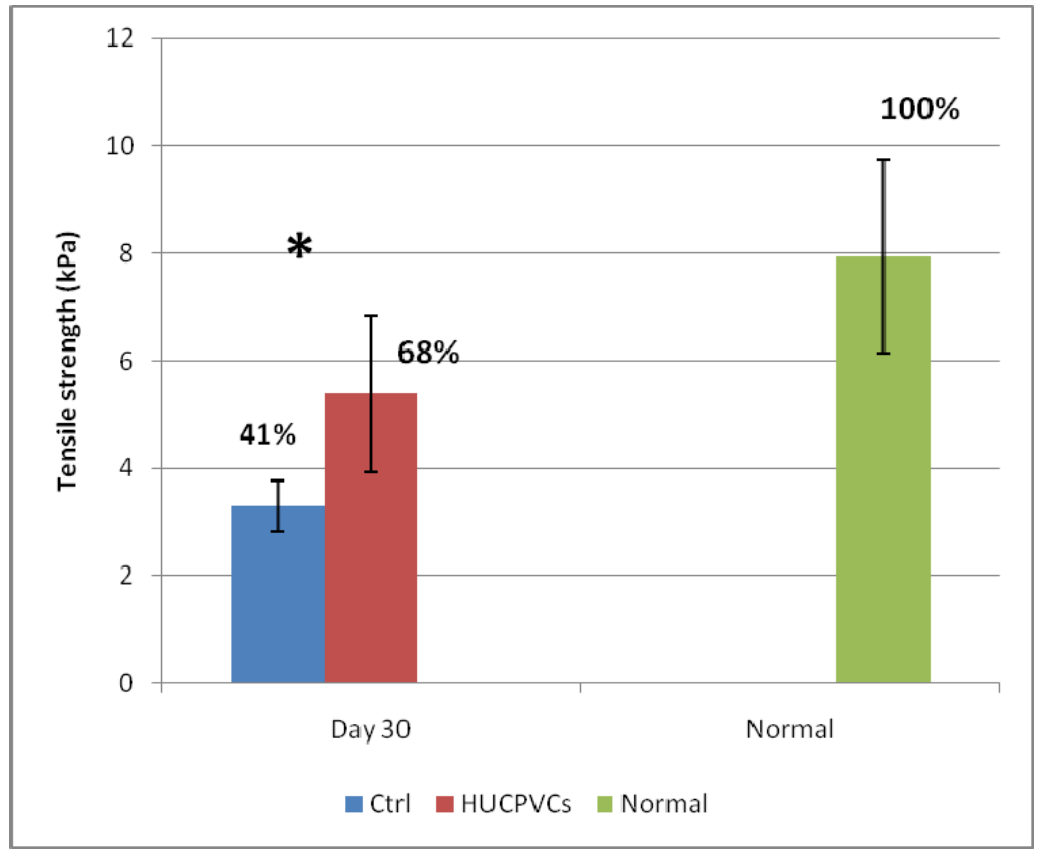

Fig. (6). Tensile strength values at 30 days post surgery compared with normal uninjured tendon. Star shows statistical significance. This HUCPVC value was $68 \%$ of the tensile strength of a normal tendon, whereas the control group was only $41 \%$. Tensile strength measurements were calculated at 7 and 15 days post surgery; however, no significant differences were seen between the treatment and the control group. 
collagen, effecting collagen fiber alignment, and improving mechanical properties when compared to sham operated controls.

\subsection{HUCPVCs Survive Xenogeneic Transplant and Express Collagen 1}

Taken together our histology, immunostaining and PCR data clearly demonstrate that HUCPVCs not only survived but contributed to the collagen organization of the repaired tendons. But, the non-nuclear specific $\mathrm{HuNu}$ labeling raises the question of whether labeled human protein had been phagocytosed by host macrophages. However, the lack of congruence in $\mathrm{HuNu}$ and $\mathrm{CD} 68$ labeling in serial sections would indicate that the two cell populations were distinct. This, together with the $\beta$-actin- 5 expression, provides robust evidence for HUCPVC survival in this model.

The change in collagen organization with time has already been reported by Williams et al., [23] who considered early scar collagen to lack crosslinking of Type I fibers and contain a higher percentage of Collagen Type III. Clearly, the presence of HUCPVCs has accelerated the change from disorganized to parallel linear collagen fiber bundles, and this observation corroborates that of Chong et al. who employed bone marrow MSCs to treat rabbit tendon injuries [8]. While the fibrin carrier used by Chong et al. could have had an influence on collagen re-organization, this was evidently not the case in our model where the cells represented the only variable between experimental and control groups.

Unfortunately, we were unable to find an anti-human collagen Type I antibody that did not cross-react with rat collagen, therefore it was not possible to determine immunohistologically if the re-organized collagen, the major protein constituent of tendon [24] was, in part, human. However, we have shown in different studies that HUCPVCs can contribute to bone [25], cartilage [14], and dermal tissue [26] in vivo, and thus it may be reasonable to expect that the collagen expression data in this study would have reflected some synthetic activity in vivo. Indeed, the change from ovoid to elongated morphology by 30 days could be indicative of tenocytic differentiation, although our primers for decorin, tenomodulin, and tenascin- $\mathrm{C}$ were unable to distinguish between the human and rat tenocyte phenotypes. Thus, we cannot assert whether the HUCPVCs differentiated into tenocytes and produced collagen, or simply acted as facilitators to increase collagen production in host tendon fibroblasts [27].

\subsection{HUCPVCs Enhance Mechanical Properties in Healing Tendon in Nude Rats}

The experimental groups at all the different time points showed an increase in mechanical strain values when compared to the control group. The Wilcoxon MatchedPairs-Signed-Rank Test showed no significance in the difference in tensile strength between the experimental and control groups at 7 and 15 days post transplantation time points, but the difference between the two groups at 30 days post surgical time point was significant $(\mathrm{P}<0.025)$. At 30 days post surgery tensile strength of the experimental group reached $68 \%$ of the tensile strength of a normal tendon, whereas that of the control group was only $41 \%$. This data is in accordance with the histological findings of difference in collagen fiber organization between the treatment and control groups.

Prior to the present study, the highest reported values of increase in wound tensile strength following cell administration was that of Hankemeier et al., [28] who created standardized central full-thickness patellar tendon defects in immunodeficient rats, filled them with human BMSC in a fibrin matrix, and showed $51.5 \%$ of normal tensile strength 20 days after surgery. This was an improvement over the results of Young et al., [7] who repaired rabbit Achilles tendons using MSCs seeded on a biodegradable scaffold and showed $37.2 \%$ of normal tensile strength values, by 12 weeks. Chong et al. [8] who also added BMSCs to injured rabbit Achilles tendon, showed that tendon stiffness and modulus increased significantly in the treatment group at earlier time points, but there were no significant differences at later time points. In each of these referenced studies, an MSC-seeded carrier construct was employed that can integrate into the tissue; therefore, it is unclear whether the cells or the constructs were the predominant driver of healing in the injured tendons. Our work employed no carrier, yet our results compare favorably to the results of all the mentioned studies, and the mechanical properties exceed those previously reported resulting in $68 \%$ of the normal tensile strength value in our HUCPVC group.

While tensile strength has been reported to decrease with time after injury in control samples, this is due to the unorganized and haphazard orientation of the collagen fibers in the healed tendon $[1,9]$. However, there is also a decrease in tensile strength of the HUCPVC samples. Since tensile strength is measured as force/unit area, the overall decrease in tensile strength is, in part, due to an increase in the cross sectional area of the tendons that was evident at later time points. This increase in cross sectional area could be a result of increased concentration of collagen fibers and interposed cells. According to Oshiro et al., cellular and vascular content of a healing tendon tissue in rat is at its highest level around 1 month post injury [29]. This is due to the fact that larger amounts of fibrous tissue are being laid down within and around the tendon to enhance structural strength. This in turn results in an enlarged tendon that has greater structural stiff-ness but is less efficient as a spring and therefore compromises the performance of the load bearing tendon [30]. At 30 days post surgery, stiffness of the treatment group is significantly higher (1.6 fold) compared to the control group. Interestingly, both groups had significantly higher stiffness values compared to a normal tendon. This could also be due to increase in fibrous tissue inside tendons at 30 days, as mentioned above.

\section{CONCLUSION}

Our study shows that HUCPVCs can be employed in treating tendon and ligament injuries. The healing is evident through changes in: collagen organization; cell shape and orientation; and increase in mechanical properties over those of the untreated controls. All these factors could contribute 
to the healing and repair we have seen in the equine applications of such cells referred to earlier.

\section{FOOTNOTES}

a. Nunc, Thermo Fischer Scientific, 75 Panorama Creek Drive, Rochester, NY.

b. Gibco, Invitrogen, Carlsbad, CA.

c. Beckton Dickinson Labware, Franklin Lakes, NJ.

d. Sigma Chemical Inc. St. Louis, MO, USA.

e. Leica, Wetzler, Germany.

f. Reichert-Jung 1150, Depew, NY.

g. Millipore Chemicon, Billerica, USA.

h. Vector Labs, Burlingame, CA.

i. AbD Serotec, Raleigh, NC.

j. Chemicon, Billerica, USA.

k. Invitrogen, Carlsbad, CA.

1. http://www.pasteur-lille.fr/fr/recherche/biopuces/public biopuces/Formation_2009/Ambion-Tri_Reagent.pdf

m. http://www.ensembl.org

n. http://frodo.wi.mit.edu/primer3/input.htm

o. http://ifrjr.nord.univ-mrs.fr/AmplifX-Home-page

p. Model 4301, Instron Worldwide Headquarters, Norwood, MA.

\section{ACKNOWLEDGEMENT}

Supported in part by Tissue Regeneration Therapeutics (TRT) Inc

\section{DISCLOSURE}

This manuscript represents a portion of a thesis submitted by Hamideh Emrani to the faculty of Dentistry at University of Toronto as partial fulfillment of the requirements for a Master of Science degree.

Presented in the Canadian Connective Tissue Conference (CCTC), June 2010, Toronto, Ontario, Canada.

\section{ABBREVIATION}

HUCPVC $=$ Human umbilical cord perivascular cells
BMSC $=$ Bone marrow stem or stromal cells
MSC $=$ Mesenchymal stromal cells
CFU-F $=$ Colony forming units-fibroblasts
$\mathrm{HuNu}=$ Anti human nuclei antibody

\section{REFERENCES}

[1] Sharma P, Maffulli N. Biology of tendon injury: healing, modeling and remodeling. J Musculoskelet Neuronal Interact 2006; 6(2): 181-90.
Guest DJ, Smith MRW, Allen WR. Monitoring the fate of autologous and allogeneic mesenchymal progenitor cells injected into the superficial digital flexor tendon of horses: preliminary study. Equine Vet J 2008; 40(2): 178-81.

[3] Vidal MA, Kilroy GE, Lopez MJ, et al. Characterization of equine adipose tissue-derived stromal cells: adipogenic and osteogenic capacity and comparison with bone marrow-derived mesenchymal stromal cells. Vet Surg 2007; 36(7): 613-22.

[4] Koch TG, Heerkens T, Thomsen PD, et al. Isolation of mesenchymal stem cells from equine umbilical cord blood. BMC Biotechnol 2007; 7: 26.

[5] Frisbie DD, Smith RKW. Clinical update on the use of mesenchymal stem cells in equine orthopaedics. Equine Vet $\mathrm{J}$ 2010; 42(1): 86-9.

[6] Smith RKW. Mesenchymal stem cell therapy for equine tendinopathy. Disabil Rehabil 2008; 30(20): 1752.

[7] Young RG, Butler DL, Weber W, et al. Use of mesenchymal stem cells in a collagen matrix for achilles tendon repair. J Orthop Res 1998; 16(4): 406-13

[8] Chong AKS, Ang AD, Goh JCH, et al. Bone marrow-derived mesenchymal stem cells influence early tendon-healing in a rabbit achilles tendon model. J Bone Joint Surg Am 2007; 89(1): 74-81.

[9] Awad HA, Boivin GP, Dressler MR, et al. Repair of patella tendon injuries using a cell-collagen composite. J Orthop Res 2003; 21(3): 420-31.

[10] Hankemeier S, van Griensven M, Ezechieli M, et al. Tissue engineering of tendons and ligaments by human bone marrow stromal cells in a liquid fibrin matrix in immunodeficient rats: results of a histologic study. Arch Orthop Trauma Surg 2007; 127(9): 815-21.

[11] Sarugaser R, Lickorish D, Baksh D, et al. Human umbilical cord perivascular (HUCPV) cells: a source of mesenchymal progenitors. Stem Cells 2005; 23(2): 220-9.

[12] Schugar RC, Chirieleison SM, Wescoe KE, et al. High harvest yield, high expansion, and phenotype stability of CD146 mesenchymal stromal cells from whole primitive human umbilical cord tissue. J Biomed Biotechnol 2009; [Epub ahead (16)].

[13] Farias VA, Linares-Fernández JL, Peñalver JL, et al. Human umbilical cord stromal stem cell express CD10 and exert contractile properties. Placenta 2011; 32(1): 86-95.

[14] Sarugaser R, Hanoun L, Keating A, et al. Human mesenchymal stem cells self-renew and differentiate according to a deterministic hierarchy. PLoS ONE 2009; 4(8): e6498.

[15] Dominici M, Blanc KL, Mueller I, et al. Minimal criteria for defining multipotent mesenchymal stromal cells. Cytotherapy 2006; 8(4): 315-7.

[16] Ennis J, Götherström C, Le Blanc K, et al. In vitro immunologic properties of human umbilical cord perivascular cells. Cytotherapy 2008; 10(2): 174-81.

[17] Grinnemo KH, Månsson A, Dellgren G, et al. Xenoreactivity and engraftment of human mesenchymal stem cells transplanted into infarcted rat myocardium. J Thorac Cardiovasc Surg 2004; 127(5): 1293-300.

[18] Chen Y-J, Wang C-J, Yang KD, et al. Extracorporeal shock waves promote healing of collagenase-induced Achilles tendinitis and increase TGF-beta1 and IGF-I expression. J Orthopaed Res 2004; 22(4): 854-61.

[19] Marsolais D, Cote CH, Frenette J. Pifithrin-alpha, an inhibitor of p53 transactivation, alters the inflammatory process and delays tendon healing following acute injury. Am J Physiol Regul Integr Comp Physiol 2007; 292(1): R321-7.

[20] Williams IF, McCullagh KG, Goodship AE, Silver IA. Studies on the pathogenesis of equine tendonitis following collagenase injury. Res Vet Sci 1984; 36(3): 326-38.

[21] Shi S, Key M, Kalra K. Antigen retrieval in formalin-fixed, paraffin-embedded tissues: An enhancement method for immunohistochemical staining based on microwave oven heating of tissue sections. J Histochem Cytochem 1991; 39(6): 741-8.

[22] Emrani H. Umbilical cord perivascular cells: A mesenchymal cell source for treatment of tendon and ligament injuries. MSc thesis, University of Toronto, 2009

[23] Williams IF, Heaton A, McCullagh KG. Cell morphology and collagen types in equine tendon scar. Res Vet Sci 1980; 28(3): 302 10

[24] O'Brien M. Structure and metabolism of tendons. Scand J Med Sci Sports 1997; 7(2): 55-61. 
[25] Davies JE, Matta R, Mendes VC, et al. Development, characterization and clinical use of a biodegradable composite scaffold for bone engineering in oro-maxillo-facial surgery. Organogenesis 2010; 6(3): 161-6.

[26] Zebardast N, Lickorish D, Davies JE. Human umbilical cord perivascular cells (HUCPVC): A mesenchymal cell source for dermal wound healing. Organogenesis 2010; 6(4): 197-203.

[27] Sharma P. Tendon Injury and Tendinopathy: Healing and Repair. J Bone Joint Surg Am 2005; 87: 187-202.
[28] Hankemeier S, Hurschler C, Zeichen J, et al. Bone marrow stromal cells in a liquid fibrin matrix improve the healing process of patellar tendon window defects. Tissue Eng Part A 2009; 15(5): 1019-30.

[29] Oshiro W, Lou J, Xing X, et al. Flexor tendon healing in the rat: a histologic and gene expression study. J Hand Surg 2003; 28(5): 814-23.

[30] Richardson LE, Dudhia J, Clegg PD, et al. Stem cells in veterinary medicine-attempt at regenerating equine tendon after injury. Trends Biotechnol 2007; 25(9): 409-16.

(C) Emrani and Davies; Licensee Bentham Open.

This is an open access article licensed under the terms of the Creative Commons Attribution Non-Commercial License (http:/creativecommons.org/ licenses/by-nc/3.0/), which permits unrestricted, non-commercial use, distribution and reproduction in any medium, provided the work is properly cited. 